\title{
Diferenciación de repertorios léxicos en los nombres comunes de las plantas americanas
}

Differentiation of lexical repertoires in the common names of American plants

\section{María-Teresa Cáceres-Lorenzo \\ Universidad de Las Palmas de Gran Canaria España}

\section{Marcos Salas-Pascual}

Universidad de Las Palmas de Gran Canaria España

María-Teresa Cáceres-Lorenzo: Instituto Universitario de Análisis y Aplicaciones Textuales (IATEXT), Universidad de Las Palmas de Gran Canaria, España. | Correo electrónico: mteresa.caceres@ulpgc.es

Marcos Salas-Pascual: Instituto Universitario de Análisis y Aplicaciones Textuales (IATEXT), Universidad de Las Palmas de Gran Canaria, España. 


\section{Resumen}

En la historia del léxico que da nombre a las plantas americanas, se intuye que conviven diferentes repertorios de designaciones vernáculas. Nuestras preguntas de investigación no han sido respondidas anteriormente por la bibliografía especializada y planteamos si es posible diferenciar diacrónicamente los nombres comunes de las plantas americanas según su utilización en documentos de América o de España. Para dar respuesta a esta cuestión, se ha diseñado una investigación cuantitativa y cualitativa con fundamentación documental por medio de la búsqueda de 30 especies y sus respectivos 79 fitónimos. Los resultados nos muestran la coexistencia de varios inventarios que revelan la diversidad en la configuración de un léxico de fitónimos panhispánicos.

Palabras clave: dialectología diacrónica; corpus; americanismo léxico; fitónimos.

\section{Abstract}

In the history of the lexicon that gives name to American plants, we sense that different repertoires of vernacular designations coexist. Our research questions have not yet been answered by technical bibliography and we ask whether it is possible to differentiate diachronically the common names of American plants according to their use in documents of America or Spain. In order to answer the previous question, a quantitative and qualitative research has been designed with documentary foundations through the search of 30 species and their respective 79 plant names. The results show the coexistence of several inventories that reveal the diversity in the configuration of a lexicon of Panhispanic plant names.

Keywords: diachronic dialectology; corpus; lexical Americanism; common names of plants. 


\section{Introducción}

El descubrimiento de América produjo un intercambio de productos entre el Viejo y el Nuevo Mundo que se refleja en el léxico que aparece en los documentos de cada periodo histórico (Franconie, 2000; Frago Gracia, 2005; Mancho Duque, 2005; Marcos Marín, 2009). Uno de estos contactos se materializa durante centurias en las plantas americanas y en los nombres vernáculos, comunes o fitónimos con los que se fueron denominando (Ceballos, 1986; Turpin, 1987; Earle, 2012; Lim, 2014). De hecho, es posible encontrar ejemplos en los textos que certifican el envío de estos productos vegetales a España y las designaciones, coincidentes o no, con América: [...] cuando están bien curadas se Ilevan hasta España muchas veces (CDH, 2013: s. v.); [...] Ilevase a España para el mismo efeto (CORDIAM, 2015: 37); [...] Ilaman los nuestros [pero existe también un] nombre indio (CDH, 2013: s. v.); de la qual especia entiendo que se tiene ya e entera noticia en la mayor parte despaña, por darse en mucha parte della (Alvar Ezquera, 1997: S. v.); la raíz de este nombre es la que en las boticas de España se gasta con el nombre de [...] (CDH, 2013: s. v.). De este modo, se crea un conjunto de términos peculiares que los hablantes utilizan o conocen para nombrar las diferentes especies vegetales que emplean en la alimentación, cultivo, industria, etc. Estas palabras pueden definirse como un repertorio de designaciones vernáculas de plantas en una comunidad de hablantes.

En la actualidad, a través de la información geográfica que nos proporcionan el Diccionario de americanismos (DA, 2010) y el Diccionario de la lengua española (DRAE, 2014), y de los ejemplos textuales de los corpus (Alvar Ezquerra, 1997; Boyd Bowman, 2003; NTLE, 2007; CDH, 2013; CORDIAM, 2015), se comprueba la convivencia de diversos fitónimos que se regis-

1 Los ejemplos seleccionados son parecidos a muchos otros: Fernández de Oviedo (Santo Domingo, 1535): Cuando las batatas están bien curadas se llevan hasta España muchas veces, cuando los navios aciertan a hacer presto el viaje, pero las más veces se pierden por la mar. Yo las he llevado desde aquesta cibdad de Santo Domingo de la isla Española hasta la cibdad de Avila; y aunque no llegaron tales como acá estaban e las hay, fueron habidas por muy singular e buena fruta e se tenían en mucho; Nicolás Monardes escribe: Ilaman los nuestros Palo de las Indias, guayacan es nombre indio (1565-1574); en un texto sobre Puerto Rico (1582) se dice: [...] ay en la banda del sur desta ysla vn palo que llaman guayacan que es medeçinal para dar sudor a las bubas y otras enfermedades de frio, llevase a España para el mismo efeto y para tinir panos en flandes otro desta calidad, se llama palo santo sirve del mismo efeto. Pedro de Aguado escribe en su crónica Conquista y población de Santa Marta y Nuevo Reino de Granada (1581): La falta de sal suplía çierto género de pimienta que en las yndias o en muchas partes dellas es llamada agi, de la qual especia entiendo que se tiene ya e entera noticia en la mayor parte despaña, por darse en mucha parte della; y desde Venezuela, en el siglo XVIII, hemos encontrado en la Historia corográfica, natural y evangélica de la Nueva Andalucía (1758, Venezuela) lo que sigue: batatilla: la raíz de este nombre es la que en las boticas de España se gasta con el nombre de mechoacán o jalapa blanca. 
tran en los diccionarios como indicadores de la configuración de las distintas variedades del español panhispánico².

Estas colecciones muestran que los nombres vernáculos de las plantas americanas se incorporaron al vocabulario del conocimiento de América en las dos orillas del Atlántico, desde 1492 hasta el siglo XX (Frago Gracia, 2005; Company, 2012). Dichas plantas, con sus frutos y semillas, formaban parte de la exótica realidad americana de los que vivían en el Nuevo Mundo, pero, también, su designación y descripción pasa a tener interés en los textos, por el rédito alimentario, económico y social que representaban para la sociedad colonial y española3. Esta idea ha sido confirmada por Zamora Munné (1976: 116) y Mejías (1980: 22), quienes insisten, como resultado de una investigación con textos no literarios de los siglos XVI y XVII, respectivamente, en que el número de fitónimos es muy alto con respecto a la de otras áreas léxicas. Por su parte, Quirós García y Ramírez Luengo (2015: 197) corroboran lo anterior con documentos de Yucatán (1650-1800) en los que los nombres de las plantas son un 10\% de los americanismos, solo superados por la descripción de la organización social, industrial y la alimentación.

Desde las primeras centurias, en América los textos cronísticos informan sobre la naturaleza de las Indias a los poderes fácticos españoles. En estas obras con vocación etnográfica, la designación de una planta o su fruto puede tener un carácter erudito, pero también es un

2 A modo de ejemplos representativos, para denominar a la especie Zea mays, los diccionarios DA (2010) y DRAE (2014), así como la base documental, evidencian la coexistencia de diferentes repertorios: maíz (tainismo de uso panhispánico), que se utiliza para dar nombre a la gramínea tanto en textos españoles como americanos (Boyd Bowman, 2003; CDH, 2013); millo (occidentalismo léxico) y panizo (del español del norte peninsular), que se registran casi exclusivamente en los textos españoles (García Mouton, 1986; Cala Carvajal, 2001; Boyd Bowman, 2003; CDH, 2013); y, por último, la construcción trigo de Indias, que aparece con frecuencia desde el siglo XVI (Bravo García y Cáceres Lorenzo, 2013). En los nombres comunes de Amaranthus spp. encontramos otra tendencia similar, que refuerza la posibilidad de que existan distintos repertorios para denominar a las plantas de esta familia, ya que se emplea amaranto (voz llevada por los españoles) en textos comunes; bledo (del español), en documentos españoles, frente a dahue (del mapuche), quihuicha y quinua (quechuismos), que se utilizan en documentos americanos (DA, 2010; CDH, 2013; DRAE, 2014; CORDIAM, 2015).

3 Los textos españoles del NTLE (2007: S. v.) nos muestran cómo el nahuatlismo tomate pasa de ser un cultivo exótico (planta nueua en España) hasta convertirse en un producto español en el siglo XVIII. En 1592, el sacerdote Gregorio de los Ríos escribe en su tratado sobre la jardinería que del pomate, ay dos o tres maneras. Es vna planta que lleua pomas aquateronadas. Buélbese coloradas. No huelen. Dizen que son buenas para salsa. [...]; y Fray Diego de San José (1619) dice: tomate, es planta nueua en España. Vino de Yndias con el nombre de la tierra de donde se trajo; llámase en latín pomum aureum. La hoja es fétida, el fructo de suyo no muy mui sabroso, pero si se fríe con cebolla y quaja con huevos hace vna sabrosa cazuela que engaña pareciendo de carne. Este fruto es algo diffícil de cozer en el estómago. En Andaluzia se vsa mucho desta planta guisada y en ensalada cruda o salsa con pimiento de Yndias. 
reflejo de un uso cotidiano ${ }^{4}$. Por su parte, las Relaciones Geográficas de Indias, documentos administrativos y descriptivos que se generaron con el cuestionario de cincuenta preguntas de la Instrucción y memoria de las relaciones de que se han de hacer para la descripción de las Indias (1577), emplean para responder este interrogatorio nombres comunes de hierbas o plantas aromáticas, granos, semillas, hortalizas, árboles silvestres y de cultura propios de América, sus frutos y beneficios.

Todos estos datos aparecen en los textos a través de definiciones, enumeraciones, traducciones, sinonimias entre un vocablo indígena y español, con anotaciones que nos indican la variabilidad de posibilidades designativas y la existencia de varios repertorios (Bravo García y Cáceres Lorenzo, 2013). Asimismo, desde la perspectiva renacentista, las obras de Nicolás Monardes (1508-1588) y Francisco Hernández (1514-1578) recopilan términos con los que tratan de divulgar los conocimientos sobre la aplicación de las referidas plantas con su respectivo nombre en lengua española (Mancho Duque, 2005; López Piñero, 2007), aunque esta posible vía de incorporación de nombres vulgares a través de los textos científicos españoles parece ser muy limitada hasta el siglo XVIII (Alvar Ezquerra, 1987).

Las diferentes designaciones panhispánicas o regionales diferenciales documentadas textualmente han dado pistas a los investigadores sobre la elaboración de distintos repertorios para dar nombres a las plantas americanas, y certifican que para ello se utiliza una voz indígena o un vocablo español que sufre algún tipo de transformación semántica o morfológica (Bravo García y Cáceres Lorenzo, 2013). Durante el siglo XVII, es evidente el proceso de aceptación, regionalización y la preferencia de las comunidades hispánicas de un vocablo frente a otro, con cierta tendencia a utilizar una voz española, siempre que fuera posible (Mejías, 1980), aunque la medicina renacentista acepte las plantas americanas con sus respectivos nombres (Huguet-Termes, 2001; López Piñero, 2007).

Frago Gracia y Franco Figueroa (2003: 52) concluyen que el siglo XVIII fue una centuria muy fructífera para la llegada del indoamericanismo a España, debido a la apertura comercial y al auge de la industria farmacéutica. Ya en el siglo XIX y XX, la lexicografía española continúa la postura de incluir americanismos del Diccionario de autoridades (1726-1739) y del Diccionario castellano de Terreros y Pando (1786-1793), por lo que se registran muchos fitónimos que pueden investigarse con los atlas lingüístico y etnográfico, los diccionarios diferenciales y los corpus que hacen viable la búsqueda del testimonio

4 Las investigaciones de Galeote López (1997), Enguita Utrilla (2004), Paden (2007), Bastardín Candón (2013) y Cáceres Lorenzo (2015) explican la presencia de fitónimos en los textos cronísticos de Cristóbal Colón, Pedro Mártir de Anglería, Hernán Cortés, Fernández de Oviedo, Bernardino de Sahagún, José de Acosta, Pedro Cieza de León, entre otros. En las obras de los cronistas aparecen voces que tienen una gran vitalidad, junto a otras que apenas aparecen en los documentos (Frago Gracia y Franco Figueroa, 2003: 245). 
textual (Alvar Ezquerra, 1987; Corrales, 2004; García Mouton, 2009; Arias Álvarez y Hernández Mendoza, 2013).

A tenor de lo anterior, en el presente trabajo buscamos el apoyo documental de los fitónimos, con el fin de verificar la presunta utilización de varios inventarios de nombres comunes para las plantas americanas. Las preguntas de investigación que nos planteamos son las siguientes: ¿es posible diferenciar diacrónicamente los nombres comunes de las plantas americanas según su utilización en documentos de América o de España?; ¿los fitónimos usados en textos americanos o españoles son complementarios o excluyentes?; y ¿si existieran distintos repertorios de fitónimos, es viable definir cada uno?

Creemos que la investigación diseñada para dar respuestas a estas preguntas nos proporcionará datos diacrónicos de carácter documental sobre el léxico de fitónimos empleado para designar las plantas americanas. Estas referencias pueden completarse con el análisis de otras voces que describan el mundo natural de las Indias y el proceso llevado a cabo con respecto a la creación de un vocabulario panhispánico y diferencial.

\section{Material y método}

Con el propósito de dar respuestas a las preguntas anteriores, se ha diseñado una investigación que tiene las fases que siguen:

a) Elaboración de una base de datos en la que aparecen las especies vegetales y sus respectivos nombres comunes, según se registra en DA (2010) y DRAE (2014), tal como se refleja en la tabla 1. En la selección de estos nombres, se han tenido en cuenta que fueran fitónimos con ejemplos en los documentos y que respondieran a una de tres posibilidades, según las marcas geográficas: i) una difusión general en el español europeo; ii) la presencia léxica en varios países de América; y iii) una propagación común panhispánica5.

b) Para el cálculo de la frecuencia de uso de estos términos, así como para valorar la distribución geográfica de los mismos, se ha empleado el Corpus del nuevo diccionario histórico del español (CDH, 2013). Dicho corpus se compone de 355.740.238 registros con textos del español panhispánico, que incluyen la información de otras obras académicas: Nuevo diccio-

5 La aplicación de estos criterios presenta la posibilidad de que en varios casos no se refiera a una única especie, sino a varias especies de un mismo género, este es el caso de los géneros Opuntia, Agave, Cucurbita, Passiflora, Amaranthus y Yucca, géneros ampliamente cultivados, con multitud de variedades y cultivares, que, en la mayor parte de los casos, se designan con nombres genéricos o con nombres compuestos a partir del más general, por ejemplo, nopal y nopal cardón. De ahí la imposibilidad de ligar a una única especie los fitónimos utilizados en estos casos. 
nario histórico del español, más los datos de Corpus diacrónico del español y del Corpus de referencia del español actual. La suma de textos de todas las áreas hispánicas que ofrece es superior a la de otros corpus como Alvar Ezquerra (1997), Boyd Bowman (2003), NTLE (2007) y CORDIAM (2015), que también usaremos para un análisis de los primeros resultados obtenidos en DA (2010) y DRAE (2014).

El CDH (2013) permite conocer el número de veces en que los diferentes textos utilizan un determinado fitónimo, así como las obras y las zonas lingüísticas en las que se publicaron. Este dato puede utilizarse como una frecuencia de uso, aunque con determinadas limitaciones; la principal es que no discrimina el significado de ciertos términos. Un ejemplo de lo que exponemos es el fitónimo piña, ya que no pueden diferenciarse los ejemplos en que se refiere a las piñas de un pino o al fruto de Ananas comosa, por lo que los datos que nos ofrece no son pertinentes para nuestra investigación y, por ende, no se utilizan para la misma.

Otra limitación del CDH es que considera como referencia geográfica el lugar de publicación de la obra donde aparece el término buscado. Esto implica que aquellos textos que tratan de cuestiones americanas, pero que se publicaron en España, se indican con esta última marca geográfica. Esto es muy frecuente durante los siglos XV y XVI con relación a las obras americanas, porque la imprenta no había llegado a todas las ciudades del Nuevo Mundo (Nájera, 2012). Para solventar en parte este problema, hemos realizado los cálculos considerando únicamente los textos editados a partir de 1600, intentando minimizar este efecto comentado. Aun así, son muchos los textos de los siglos XVII y XVIII que se publican en España y que versan sobre cosas de América y que, por tanto, utilizan estos términos, sin que sean ejemplos de su uso mayoritario en el español europeo. Aunque no sea posible eliminar del todo este problema, el conocimiento del mismo nos permite minimizar su efecto sobre el análisis de los datos obtenidos a través de la consulta manual.

Y, por último, existe una desproporción significativa entre el número de textos de cada región que se incluyen en el $\mathrm{CDH}$. El número de referencias o casos encontrados en la base, entre los años 1600 y 2000 es de 316.086.115, de los que 212.945 .136 se refieren a España y 103.140.979 a zonas de América. Para poder utilizar los datos obtenidos del CDH sin que esta desproporción influya en los mismos, se ha calculado un índice de corrección que nos permitirá conocer el número de apariciones de un término si existiese igual número de casos americanos que españoles (158.043.058). Este índice se calcula con la siguiente fórmula:

\section{número de términos ponderado $=\frac{158.043 .058 \times \text { número de términos en el CDH }}{\text { número de casos en el CDH }}$}

Para los fitónimos americanos, el número ponderado se obtiene de multiplicar el obtenido en el CDH por 1,53, mientras que para los españoles el valor corregido se calcula tras multiplicar 
el dato extraído del CDH por 0,74. Aún así, el valor no es exactamente proporcional, ya que para serlo debería relacionarse con un valor objetivo, como el número de hablantes de cada zona lingüística. Pero la dificultad de conocer este valor desde un punto de vista histórico haría imposible el análisis. Con el método escogido para esta investigación, es posible obtener no una frecuencia de uso real precisa, sino un valor relacionado con la misma, que nos permite diferenciar esta variable en diferentes regiones hispanohablantes.

Con los datos ponderados de esta manera, la proporción entre los valores de aparición de cada término nos indicará si se trata de un nombre vernáculo utilizado mayoritariamente en una u otra región, o en ambas. Se entenderá que un fitónimo pertenece al repertorio americano cuando la proporción entre apariciones en textos americanos y españoles sea inferior a 0,12, es decir, un fitónimo recogido en España por cada 8 presentes en textos americanos. Aquellos que alcanzan valores intermedios, entre 0,12 y 1,12, vale decir, entre 1 de cada 8 y 9 de cada 8, se consideran fitónimos presentes en ambos, mientras que aquellos con una proporción superior a 1,12 se consideran propios del español.

Es evidente que estos límites no deben considerarse inmutables, ya que es muy difícil determinar con precisión cuándo un término pertenece a un repertorio determinado. En nuestro caso, los fitónimos que alcanzan valores cercanos a estos límites propuestos deben considerarse como de dudosa ubicación en un inventario concreto. Una vez estudiada la existencia de nombres de plantas distintos en América y España, se procederá a realizar un estudio histórico de la creación de estos. Para ello, se emplean las referencias temporales que aparecen en el CDH (2013), así como en diversas obras clásicas sobre la historia del español.

\section{Resultados}

La planificación para obtener resultados que respondan a las preguntas de investigación nos proporciona 30 especies o géneros con sus respectivos 79 fitónimos, que aparecen en la tabla 1.

\section{TABLA 1}

Especies y fitónimos seleccionados

\begin{tabular}{ll}
\multicolumn{1}{c}{ ESPECIES } & \multicolumn{1}{c}{ FITÓNIMOS } \\
Acacia farnesiana & aromo, huizache \\
\hline Agave spp. & cabuya, maguey, pita, pitera \\
\hline Amaranthus spp. & amaranto, bledo, quihuicha, quinua \\
\hline Ananas comosus & ananás, piña, piña tropical, piña de indias, piña de América \\
\hline Annona cherimola & chirimoya \\
\hline Arachis hypogaea & cacahuate, cacahuete, maní \\
\hline
\end{tabular}




\begin{tabular}{|c|c|}
\hline Bougainvillea glabra & buganvilla, trinitaria, veranera \\
\hline Bursera graveolens & caraña \\
\hline Capsicum grex. annuum & ají, chile, guindilla, pimiento-a \\
\hline Carica papaya & papaya-o, papayero \\
\hline Casimiroa edulis & zapote \\
\hline Cucurbita spp. & auyama, calabaza, calabacera, mate, porongo, tapara, zapallo \\
\hline Guayacum officinale & guayacán, palo santo, palo de las indias \\
\hline Helianthus annua & girasol \\
\hline Ipomoea batatas & batata, boniato, camote \\
\hline Lycopersicum sculentum & jitomate, tomate \\
\hline Mammea americana & mamey \\
\hline Manihot esculenta & yuca \\
\hline Mirabilis jalapa & dondiego, jalapa \\
\hline Opuntia spp. & chumbera, higo chumbo, nopal, tuna-o \\
\hline Passiflora spp. & granadilla, flor o fruto de la pasión, maracuyá, parchita \\
\hline Persea americana & aguacate, curo, palta \\
\hline Phaseolus vulgaris & caraotas, frijol, judía, poroto \\
\hline Psidium guajava & guayaba-o \\
\hline Smilax medica & zarzaparrilla \\
\hline Solanum tuberosum & papa, patata \\
\hline Tagetes patula & clavelón \\
\hline Theobroma cacao & cacao \\
\hline Yucca spp. & izote, yuca \\
\hline Zea mays & maíz, millo, panizo, trigo de indias \\
\hline
\end{tabular}

Los valores obtenidos tras el tratamiento de los fitónimos analizados mediante los datos ponderados encontrados en el CDH se muestran en la tabla 2.

\section{TABLA 2}

Resultados numéricos

\begin{tabular}{lcccccl} 
FITÓNIMOS & $\begin{array}{c}\text { CASOS EN } \\
\text { ESPAÑA }\end{array}$ & $\begin{array}{c}\text { CASOS EN } \\
\text { AMÉRICA }\end{array}$ & $\begin{array}{c}\text { VALORES } \\
\text { CORREGIDOS } \\
\text { ESPAÑA }\end{array}$ & $\begin{array}{c}\text { VALORES } \\
\text { CORREGIDOS } \\
\text { AMÉRICA }\end{array}$ & PROPORCIÓN & REPERTORIO \\
aguacate & 142 & 540 & 105,39 & 827,44 & 0,13 & común \\
\hline ají & 93 & 818 & 69,02 & 1253,42 & 0,06 & americano \\
\hline
\end{tabular}




\begin{tabular}{|c|c|c|c|c|c|c|}
\hline amaranto & 121 & 263 & 89,80 & 403,00 & 0,22 & común \\
\hline ananás & 31 & 31 & 23,01 & 47,50 & 0,48 & común \\
\hline aromo & 25 & 71 & 18,55 & 113,22 & 0,16 & común \\
\hline auyama & 10 & 43 & 7,42 & 65,89 & 0,11 & americano \\
\hline batata & 180 & 397 & 133,59 & 608,32 & 0,22 & común \\
\hline bledo & 221 & 131 & 164,02 & 200,73 & 0,82 & común \\
\hline boniato & 75 & 97 & 55,66 & 148,63 & 0,37 & común \\
\hline buganvilla & 92 & 37 & 68,28 & 56,70 & 1,20 & español \\
\hline cabuya & 17 & 183 & 12,62 & 280,41 & 0,04 & americano \\
\hline cacahuate & 2 & 113 & 1,48 & 173,15 & 0,01 & americano \\
\hline cacahuete & 256 & 48 & 190,00 & 73,55 & 2,58 & español \\
\hline cacao & 779 & 1509 & 578,16 & 2312,24 & 0,25 & común \\
\hline calabacera & 18 & 0 & 13,36 & 0,00 & $\infty$ & español \\
\hline calabaza & 1359 & 989 & 1008,62 & 1515,45 & 0,67 & común \\
\hline camote & 40 & 296 & 29,69 & 453,56 & 0,07 & americano \\
\hline caraña & 18 & 6 & 13,36 & 9,19 & 1,45 & español \\
\hline caraotos & 0 & 67 & 0,00 & 102,66 & 0,00 & americano \\
\hline chile & 33 & 604 & 24,49 & 925,51 & 0,03 & americano \\
\hline chirimoya & 51 & 105 & 37,85 & 160,89 & 0,24 & común \\
\hline chumbera & 124 & 8 & 92,03 & 12,26 & 7,51 & español \\
\hline clavelón & 16 & 4 & 11,87 & 6,13 & 1,94 & español \\
\hline curo & 0 & 0 & 0 & 0 & 0 & americano* \\
\hline dondiego & 51 & 3 & 37,85 & 4,60 & 8,23 & español \\
\hline flor de la pasión & 14 & 5 & 10,39 & 7,66 & 1,36 & español \\
\hline frijol & 148 & 1387 & 109,84 & 2125,30 & 0,05 & americano \\
\hline girasol & 354 & 294 & 262,73 & 450,50 & 0,58 & común \\
\hline granadilla & 7 & 27 & 5,20 & 41,37 & 0,13 & americano \\
\hline guayaba-o & 94 & 623 & 69,76 & 954,62 & 0,07 & americano \\
\hline guayacán & 43 & 174 & 31,91 & 266,62 & 0,12 & americano \\
\hline guindilla & 330 & 16 & 244,92 & 24,52 & 9,99 & español \\
\hline henequén & 6 & 83 & 4,45 & 127,18 & 0,04 & americano \\
\hline higo chumbo & 35 & 14 & 25,98 & 21,45 & 1,21 & español \\
\hline huizache & 0 & 59 & 0,00 & 90,41 & 0,00 & americano \\
\hline
\end{tabular}




\begin{tabular}{|c|c|c|c|c|c|c|}
\hline izote & 0 & 11 & 0,00 & 16,86 & 0,00 & americano \\
\hline jalapa & 28 & 10 & 20,78 & 15,32 & 1,36 & español \\
\hline jitomate & 3 & 606 & 2,23 & 928,57 & 0,00 & americano \\
\hline judía & 0 & 0 & 0 & 0 & 0 & español* \\
\hline maguey & 96 & 344 & 71,25 & 527,11 & 0,14 & americano \\
\hline maíz & 2417 & 5793 & 1793,84 & 8876,62 & 0,20 & común \\
\hline mamey & 41 & 204 & 30,43 & 312,59 & 0,10 & americano \\
\hline maní & 109 & 558 & 80,90 & 855,02 & 0,09 & americano \\
\hline maracuyá & 6 & 18 & 4,45 & 27,58 & 0,16 & común \\
\hline mate & 0 & 0 & 0 & 0 & 0 & americano** \\
\hline millo & 57 & 58 & 42,30 & 88,87 & 0,48 & común \\
\hline nopal & 78 & 198 & 59,94 & 359,55 & 0,17 & común \\
\hline palo de (las) Indias & 20 & 0 & 14,84 & 0,00 & $\infty$ & español \\
\hline palo santo & 137 & 80 & 101,68 & 122,58 & 0,83 & común \\
\hline palta & 52 & 278 & 38,59 & 425,98 & 0,09 & americano \\
\hline panizo & 120 & 16 & 89,06 & 24,52 & 3,63 & español \\
\hline рара & 0 & 0 & 0 & 0 & 0 & americano*** \\
\hline papaya & 103 & 385 & 76,44 & 589,94 & 0,13 & común \\
\hline papayero & 2 & 14 & 1,48 & 21,45 & 0,07 & americano \\
\hline parchita & 1 & 9 & 0,74 & 13,79 & 0,05 & americano \\
\hline patata & 5029 & 206 & 3732,41 & 315,65 & 11,82 & español \\
\hline pimiento-a & 2110 & 452 & 1565,99 & 692,60 & 2,26 & español \\
\hline piña & 1308 & 914 & 970,77 & 1400,52 & 0,69 & común \\
\hline piña americana & 7 & 1 & 5,20 & 1,53 & 3,39 & español \\
\hline piña de (las) Indias & 2 & 0 & 1,48 & 0,00 & $\infty$ & español \\
\hline piña tropical & 3 & 0 & 2,23 & 0,00 & $\infty$ & español \\
\hline pita & 191 & 247 & 141,76 & 378,48 & 0,37 & común \\
\hline pitera & 17 & 0 & 12,62 & 0,00 & $\infty$ & español \\
\hline porongo & 0 & 1 & 0,00 & 1,53 & 0,00 & americano \\
\hline poroto & 18 & 460 & 13,36 & 704,86 & 0,02 & americano \\
\hline quihuicha & 0 & 11 & 0,00 & 16,86 & 0,00 & americano \\
\hline quinua & 110 & 245 & 81,64 & 375,41 & 0,22 & común \\
\hline tapara & 0 & 0 & 0 & 0 & 0 & americano* \\
\hline
\end{tabular}




\begin{tabular}{lcccccc}
\hline tomate & 4136 & 2007 & 3069,65 & 3075,33 & 1,00 & común \\
\hline trigo de Indias & 15 & 1 & 11,13 & 1,53 & 7,27 & español \\
\hline trinitaria & 1 & 18 & 0,74 & 27,58 & 0,03 & americano \\
\hline tuna-o & 0 & 0 & 0 & 0 & 0 & americano** \\
\hline tutuma & 1 & 38 & 0,74 & 58,23 & 0,01 & americano \\
\hline veranera & 0 & 7 & 0,00 & 10,73 & 0,00 & americano \\
\hline yuca(Mandioca) & 119 & 757 & 88,32 & 1159,95 & 0,08 & americano \\
\hline yuca(Yucca) & 19 & 10 & 14,10 & 15,32 & 0,92 & común \\
\hline zapallo(II)o & 12 & 476 & 8,91 & 729,38 & 0,01 & americano \\
\hline zapote & 43 & 242 & 31,91 & 370,82 & 0,09 & americano \\
\hline zarzaparrilla & 147 & 97 & 109,10 & 148,63 & 0,73 & común \\
\hline
\end{tabular}

Los fitónimos en los que nos ha sido imposible determinar su ubicación en un repertorio o en otro mediante el análisis de sus apariciones en el CDH son aquellos términos que pueden confundirse con otras palabras de significado distinto: papa, tuna(o), tapara, mate, judía y curo. De estas últimas, tapara y curo son clasificadas como americanismos tanto por el DRAE como por el DA (indicados en la tabla 2 como americanos*); mate y tuna son consignados también por el DA, aunque el DRAE no asigna marca geográfica a estos términos (se han señalado en la tabla 2 como americanos**).

En los casos de papa y judía se ha procedido de la siguiente forma: en papa es bien conocido su uso en toda América y en las hablas meridionales, aunque en el repertorio español peninsular es ampliamente mayoritaria la designación de patata (DCECH, 2000: s. v.), por lo que se ha señalado en la tabla 3 como americanismo***; lo contrario ocurre con judía, término empleado con el sentido de nombre de planta casi exclusivamente en el español peninsular, sustituido por frijol, poroto u otras formas más locales en los textos americanos (DCECH, 2000: s. v.), por lo que se ha indicado como español*.

De esta manera, se configuran tres grupos diferentes de fitónimos que hemos denominado repertorios americanos, español y común, constituidos por 35, 21 y 23 vocablos vernáculos, respectivamente. De cada fitónimo se ha buscado su fecha de aparición en los textos de sus respectivas zonas de uso y se ha obtenido la tabla 3.

Tal como se aprecia, el 60\% de los vocablos son indigenismos, con 48 términos. Destacando, en este sentido, los ejemplos encontrados en el repertorio americano, donde aparecen 30 ejemplos, lo que representa el 85,7\% del mismo. 


\section{TABLA 3}

Datos sobre los repertorios de fitónimos

\begin{tabular}{ll|ll|ll}
\hline \multicolumn{7}{c}{} & \multicolumn{4}{c}{ AMÉRICA } & 1916 & trinitaria & 1702 \\
\hline ají & 1492 & huizache & 1916 & tutuma & 1629 \\
\hline cabuya & 1573 & izote & 1743 & veranera & 1943 \\
\hline cacahuate & 1526 & jitomate & 1519 & yuca(Mandioca) & 1495 \\
\hline camote & 1521 & maguey & 1526 & zapallo & 1595 \\
\hline caraota & 1527 & mamey & 1495 & zapote & 1527 \\
\hline chile & 1687 & maní & 1553 & curo & desc. \\
\hline frijol & 1527 & palta & 1896 & tapara & desc. \\
\hline granadilla & 1519 & papayera & 1815 & mate & 1570 \\
\hline guayaba-0 & 1553 & parchita & 1589 & tuna-o & 1526 \\
\hline guayacán & 1526 & porongo & 1568 & papa & 1550 \\
\hline henequén & 1526 & poroto & 1992 & &
\end{tabular}

ESPAÑA

\begin{tabular}{ll|ll|ll} 
buganvilla & 1896 & flor(fruta)dela pasión & 1710 & piña americana) & 1896 \\
\hline cacahuete & 1793 & guindilla & 1793 & piñade(las) Indias & 1537 \\
\hline calabacera & 1852 & higo chumbo & 1828 & piña tropical & 1956 \\
\hline caraña & 1577 & jalapa & 1597 & pitera & 1872 \\
\hline chumbera & 1896 & panizo & 1527 & trigo de Indias & 1585 \\
\hline clavelón & 1847 & patata & 1793 & judía & 1535 \\
\hline dondiego & 1893 & pimiento(a) & 1492 & palo de (las) Indias & 1511
\end{tabular}

\begin{tabular}{ll|ll|ll}
\multicolumn{7}{c}{} & \multicolumn{5}{c}{ COMŨN } & 1653 & pita & 1580 \\
\hline aguacate & 1527 & chirimoya & 1535 & quinua & 1551 \\
\hline amaranto & 1793 & girasol & 1495 & tomate & 1541 \\
\hline aromo & 1578 & maíz & 1971 & yuca(Yucca) & 1793 \\
\hline batata & 1710 & maracuyá & 1580 & palo santo & 1526 \\
\hline boniato & 1519 & millo & 1553 & bledo & 1492 \\
\hline cacao & 1568 & nopal & 1535 & maguey & 1519 \\
\hline calabaza & 1519 & papaya-o & 1535 & zarzaparrilla & 1536 \\
\hline
\end{tabular}


Del análisis de la tabla 3 se extraen referencias diferenciadoras de los tres inventarios. El reparto de los diferentes términos de cada inventario en estas épocas queda reflejado en la figura 1. Téngase en cuenta que entre los fitónimos utilizados en América existen 2 de los que desconocemos su primera referencia (tapara y curo), por lo que se ha optado por incluirlos en el segundo grupo, es decir, en el que se registran los fitónimos entre los siglos XVIII y XX.

\section{FIGURA 1}

Distribución por siglos de los fitónimos en los tres repertorios

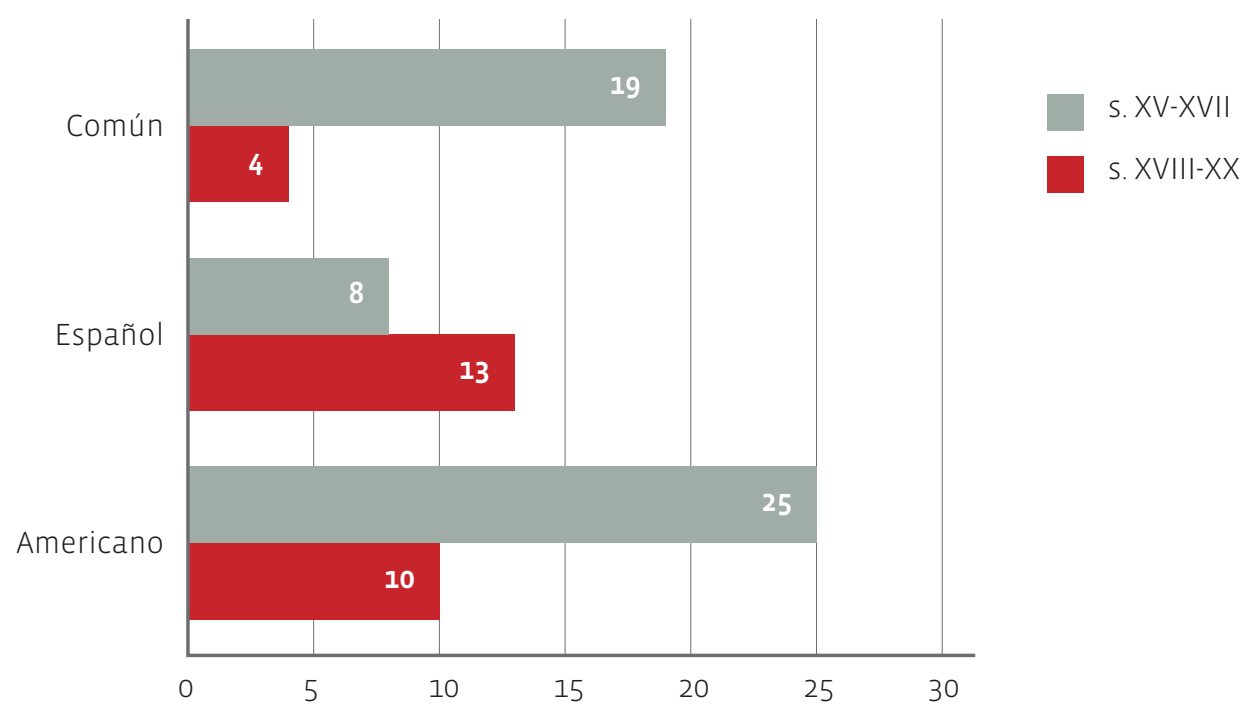

Con la cronología de los ejemplos documentales de cada fitónimo, es posible establecer dos períodos de aparición de los términos analizados en los textos: por un lado, los primeros siglos de la conquista y colonización de América, s. XV al XVII, que representan el 65,8\% (52 referencias), y, por otro, las centurias posteriores, del s. XVIII al XX, con el 34,2\% (27 ejemplos).

\section{Discusión}

La existencia de tres vocabularios o repertorios diferenciados entre los fitónimos analizados en este trabajo queda bien delimitada, tanto por los datos de uso que se presentan como por las diferentes características que poseen.

Por un lado, el conjunto de nombres vernáculos que forman el americano, integrado por 35 ejemplos textuales, con una baja frecuencia de uso, alrededor de 240 referencias por fitónimo, y caracterizado por la riqueza de indigenismos léxicos, muchos de ellos locales (Turpin, 1987). Estos términos han ido apareciendo paulatinamente en los textos, aunque son más numerosos los incorporados en los inicios de la colonización europea de América (CDH, 2013). Voces como ají, papa, cabuya, camote, yuca, tuna, zapote, etc., ya están presentes en los pri- 
meros textos que describen la naturaleza americana, y ni aun así han podido incorporarse al repertorio peninsular, a pesar de que muchas de estas plantas se cultivaron en España muy pronto, tras los primeros contactos (Alvar Ezquerra, 1997; DCECH, 2000; Franconie, 2000).

El repertorio común es el más antiguo, ya que más del 85\% de sus voces se encuentran en el vocabulario hispano desde los siglos XV al XVII, y también es el más utilizado, con más de 30.000 referencias en la base de datos empleada en este trabajo, lo que implica una media de 1.280 referencias por fitónimo. Son términos mayoritariamente indígenas, pero no en la proporción del grupo anterior. Pertenecen a este glosario las voces más usadas en todo el ámbito hispanohablante para designar a las plantas más empleadas: maíz, batata, calabaza, girasol, piña, aguacate, cacao, papaya, tomate, etc. En este repertorio se registra boniato y millo, nombres frecuentes en el área del Caribe, el primero por ser el lugar de origen de dicho término y el segundo por la existencia del fitónimo millo cubano, que denomina a una especie de sorgo forrajero, Sorghum bicolor, y no al maíz, Zea mays. Sin esta presencia en las islas antillanas, ambos fitónimos pertenecerían al vocabulario español, donde son más frecuentes (CDH, 2013: S. v.).

El tercer repertorio es el característico del español europeo. Se trata de un vocabulario muy definido, con 21 fitónimos entre los que solo existen 3 indigenismos, con una frecuencia de uso de unas 435 referencias por término, y que aparecen en los textos entre los siglos XVIII y XX. Los nombres que se emplean en España para denominar a las plantas americanas son mayoritariamente voces creadas a partir del español y que sustituyen a los fitónimos indígenas. La forma en que se produce este vocabulario se repite en muchos casos y puede resumirse en la siguiente secuencia: i) la planta americana es citada en los textos coloniales utilizando su nombre indígena o un término español relacionado; ii) el producto vegetal, junto con su nombre, es transportado hasta la Península, donde, si se aclimata, se inicia su cultivo. En la mayoría de las ocasiones el nombre que tenía en América, ya fuera indígena o castellano, es sustituido por uno local de nueva creación.

Esto ocurrió con el indigenismo nopal, que fue sustituido por chumbera, igual que su fruto, que pasó a denominarse higo chumbo (Enguita Utrilla, 2004: 58); ají o chile, que se nominó desde mediados del siglo XVI como pimiento y más tarde como guindilla (Boyd Bowman, 2003; CDH, 2013); e incluso con maíz, que tuvo varias designaciones, como panizo o trigo de Indias, y que en otras lenguas peninsulares perdió su nombre indígena para denominarse millo en gallego-portugués o panís en catalán (García Mouton, 1986; Cala Carvajal, 2001). Otro indigenismo, papa, fue mucho más tarde sustituido por patata, término creado por confusión con batata. Las citas de patata desde 1519 hasta el siglo XVIII se refieren a Ipomaea batata, y no fue hasta el periodo ilustrado cuando queda clara la designación de patata para los tubérculos de Solanum tuberosum (DCECH: S. v.). Para entonces, batata no se usaba en la gran parte de España, sustituido por boniato, lo cual impedía la confusión. Pero también los fitónimos americanos de origen español se vieron sustituidos, como ocurrió con frijol, que muy pronto se reemplazó por el término judía (CDH, 2013). 
En algunos casos, la castellanización se produjo añadiéndoles el sufijo -era, como pitera (Boyd Bowman, 2003). Otras creaciones peninsulares más modernas son las que dan nombre a muchas plantas ornamentales de origen americano: buganvilla, clavelón, dondiego, flor o fruta de la pasión (DRAE, 2014), así como también las formaciones con piña, que intentaban romper la confusión que producía la designación de esta fruta americana: piña tropical, piña de América, piña de Indias (CDH, 2013; DRAE, 2014). La reticencia del español a hacer uso de términos indígenas es muy conocida (Zamora Munné, 1976; Mejías, 1980) y puede leerse en muchos textos de los primeros momentos de la conquista (Enguita Utrilla, 2004).

Como vemos, gran parte de la razón de la formación del repertorio peninsular hay que buscarla en la presencia física de estas plantas en el territorio. Las que son conocidas en gran parte de España pero que no se cultivan en ella o lo hacen de forma puntual — chirimoyas, papayo, maracuyá, aguacate, cacao, guayabo, mamey y yuca- mantienen nombres indígenas y los diccionarios los registran como parte del acervo común del español, mientras que los cultivos muy extendidos en España, ya sea con fines alimenticios u ornamentales - pitera, buganvilla, chumbera, pimiento, dondiego, flor de la pasión, clavelón, patata y judía - adquirieron nombres propios y diferenciados de los fitónimos americanos.

Las excepciones a lo anterior son tomate y girasol, cultivos muy importantes en territorio peninsular que han mantenido su denominación hasta hoy. El primero de origen nahua y el segundo una creación castellana muy temprana. Los casos de los fitónimos caraña, zarzaparrilla y jalapa han seguido caminos similares. Bursera graveolens, la caraña, fue muy importante en los primeros inicios de la Conquista por su uso medicinal, pero nunca fue cultivada en España. Los datos muestran que caraña pertenece al vocabulario vernáculo en la medicina del Renacimiento, hoy ya en desuso (Galeote López, 1997; Huguet-Termes, 2001). El nombre del árbol del que procede la caraña aparece en el DRAE (2014) como americanismo exclusivo de Guatemala, y el DA (2010) lo indica como dialectalismo de Panamá, Colombia y Nicaragua. En este caso, se trata, pues, de una voz que en sus dos significados pertenece a repertorios distintos: como resina medicinal se incluye en el vocabulario español, y como designación regional de un árbol es un americanismo léxico.

Mirabilis jalapa, la jalapa, y Smilax medica, la zarzaparrilla, se conocen, al igual que la caraña, desde los inicios de la colonización europea de América, ya que fueron muy conocidas en la farmacopea española. Las raíces de la primera se denominaban jalapa, quizá como referencia al lugar de procedencia de la planta, mientras que la segunda se conoció como zarzaparrilla, de la misma manera que otras especies mediterráneas del mismo género (DCECH: s. v.). La jalapa se cultiva desde hace mucho tiempo en España como ornamental, pero hoy recibe el nombre de dondiego (Ceballos, 1986; Lim, 2014). Caraña, zarzaparrilla y jalapa fueron fitónimos muy frecuentes en los siglos XVI y XVII, cuando su comercio aportaba beneficios económicos, políticos y sociales. 


\section{Conclusiones}

Finalmente, se concluye que esta investigación ha demostrado la convivencia de tres repertorios de fitónimos que muestran la tendencia pluricéntrica de la norma panhispánica: el repertorio americano, formado por fitónimos de escaso uso, mayoritariamente de origen indígena y asimilados, por lo general, en los primeros siglos de la conquista; el español, integrado por términos de mayor frecuencia que los anteriores, aparecidos durante los siglos XVII al XX y con muy escasos indigenismos; y el común, de gran uso y elevado porcentaje de indigenismos, creado durante las primeras centurias del contacto.

A la cuestión que nos planteamos sobre si estos repertorios son contrapuestos o complementarios, es decir, si cada especie recibe nombres distintos en cada una de las grandes regiones de habla hispana, los resultados muestran que, de las 30 especies o géneros analizados, solo se produce una clara dicotomía entre su nombre americano y peninsular en los casos que siguen: papa/patata, buganvilla/trinitaria/veranera, aji/chile/pimiento/guindilla, y maní/cacahuate/cacahuete. La preferencia más generalizada es la de denominar a cada planta americana con un término común, al mismo tiempo que mantiene designaciones americanas o españolas minoritarias: nopal con tuna o chumbera; maracuyá con parchita o flor de la pasión; maíz con panizo; yuca con izote; batata con camote; quinua con quihuicha, etc. La generalización del cultivo y el comercio de estos vegetales americanos fomentan en la actualidad esta predisposición, por lo que es muy probable que en pocos años fitónimos exclusivos del repertorio americano como yuca 'mandioca', zapote o guayabo puedan pasar a formar parte del vocabulario panhispánico.

El proceso diacrónico de la configuración de las voces vernáculas que nombran a las plantas americanas parece haber sido más intenso en los primeros siglos coloniales, aunque se evidencia una excepción en los textos españoles del periodo ilustrado. La preferencia de los hablantes peninsulares por voces españolas frente a las indígenas o a la inversa en distintos repertorios depende de factores extralingüísticos (espacio geográfico, centuria colonial o no, expectativas sociales, intenciones comunicativas, conocimientos o experiencias comunitarias, etc.) que quedan pendientes para futuros estudios. Además, nuestros resultados deben completarse con ejemplos documentales de otras áreas léxicas (zoónimos, ictiónimos, etc.), con el fin de descubrir cómo se conforman en la lengua española las designaciones de la realidad americana.

\section{Bibliografía citada}

Alvar EzquerRa, Manuel, 1987: "La recepción de americanismos en los diccionarios generales de la lengua" en M. Tránsito Vaquero de Ramirez (ed.): Actas del I Congreso Internacional sobre el español de América, San Juan de Puerto Rico: Academia Puertorriqueña de la Lengua Espa- 
ñola, 209-218.

Alvar Ezquerra, Manuel, 1997: Vocabulario de indigenismos en las crónicas de Indias, Madrid: Consejo Superior de Investigaciones Científicas.

Arias Álvarez, Beatriz, y Juan Antonio Hernández Mendoza, 2013: "Importancia de la incorporación de los parámetros diastráticos y diafásicos en la elaboración del corpus electrónico del español colonial mexicano", Scriptum Digital 2, 5-20.

Bastardín Candón, Teresa, 2013: Vocabulario indígena en la Historia de fray Bernardino de Sahagún, Frankfurt: Peter Lang.

Boyd Bowman, Peter, 2003: Léxico Hispanoamericano (1493-1993), Nueva York: Hispanic Seminary of Medieval Studies.

Bravo García, Eva, y María Teresa Caceres Lorenzo, 2013: El léxico cotidiano en América a través de las Relaciones Geográficas de Indias, Frankfurt: Peter Lang.

CÁceres Lorenzo, María Teresa, 2015: "Dimensiones comunicativas del vocabulario nahua en las crónicas franciscanas: ¿indicios de mestizaje lingüístico o estrategia evangélica de Motolinía y Sahagún?”, Crítica Hispánica 37, 7-26.

Cala Carvajal, Rafael, 2001: "Las voces prehispanas cacao, cacahuete y maíz en diccionarios generales de lengua castellana, catalana e italiana del siglo XX", Boletín americanista 51, 25-41.

Ceballos Jiménez, Andrés, 1986: Diccionario ilustrado de los nombres vernáculos de las plantas en España, Madrid: Icona.

CDH: Instituto de Investigación Rafael Lapesa de la Real Academia Española, 2013: Nuevo diccionario histórico del español [http://web.frl.es/CNDHE/, fecha de consulta: 15 de octubre de 2015].

Company, Concepción, 2012: "El español del siglo XVIII. Un parteaguas lingüístico entre México y España” en Teresa García Godoy (ed.): El español del siglo XVIII. Cambios diacrónicos en el primer español moderno, Frankfurt: Peter Lang, 255-291.

CORDiam: Academia Mexicana de la Lengua, 2015: Corpus diacrónico y diatópico del español de América [www.cordiam.org, fecha de consulta: 15 de enero de 2016].

Corrales Zumbado, Cristóbal, 2004: "Causas y efectos de la lexicografía diferencial” en Cristóbal CoRRAles Zumbado y otros (coords.): Nuevas aportaciones a la historiografía lingüística, Madrid: Ed. Arco/Libros, 47-74.

DA: Asociación de Academias de la Lengua Española, 2010: Diccionario de americanismos, Madrid: Santillana.

DCECH: Joan Corominas y José Antonio Pascual, 2000: Diccionario crítico etimológico castellano 
e hispánico, Madrid: Gredos.

DRAE: Real Academia de la Lengua Española, 2014: Diccionario de la lengua española, Madrid: Espasa Calpe.

EARLE, Rebecca, 2012: The body of the conquistador. Food, race and the colonial experience in Spanish America, Cambridge: Cambridge University Press, 1492-1700.

Enguita Utrilla, José María, 2004: Para la historia de los americanismos léxicos, Frankfurt: Peter Lang.

Frago Gracia, Juan Antonio, 2005: "El indoamericanismo léxico en España. De Colón a Monardes” en Estudios ofrecidos a Antonio Quilis, Madrid: CSIC, 1719-1728.

Frago Gracia, Juan Antonio, y Mariano Franco Figueroa, 2003: El español de América, Cádiz: Universidad.

Franconie, Hélène, 2000: "Things from the new world in the European dialects", Food and Foodways 9 (1), 21-58.

Galeote López, Manuel, 1997: Léxico indígena de flora y fauna en tratados sobre las Indias Occidentales de autores andaluces, Granada: Universidad de Granada.

García Mouton, Pilar, 1986: "Justificación metodológica de un atlas lingüístico para el castellano del centro de España”, Estudis romànics 31, 263-266.

Garcia Mouton, Pilar, 2009: "Justificación metodológica de un atlas lingüístico para el castellano del centro de España”, Estudis romànics 31, 263-266.

Huguet-Termes, Terry, 2001: "New World materia medica in Spanish renaissance medicine: From scholarly reception to practical impact”, Medical History 45 (3), 359-76.

Lım, T. Keylan, 2014: "Mirabilis jalapa”, Edible Medicinal and Non-Medicinal Plants, vol. 8, Utrecht: Springer Netherlands, 497-513.

López PIÑero, José María, 2007: Medicina e historia natural en la sociedad española de los siglos XVI y XVII, Valencia: Universitat de València.

Mancho Duque, María Jesús, 2005: "La divulgación científica y sus repercusiones léxicas en la época del Quijote”, Panace@: Revista de Medicina, Lenguaje y Traducción 6 (21-22), 285-298.

Marcos Marín, Francisco, 2009: "Palabras americanas en la ciencia europea del XVI" en Luis Luque Toro (ed.): Léxico español actual II, Venezia: Università Ca` Foscari, 145-155.

Mejías, Hugo A., 1980: Préstamos de lenguas indígenas en el español americano del siglo XVII, México: UNAM. 
NÁJERA, Luna, 2012: "Contesting the Word: The Crown and the Printing Press in Colonial Spanish America", Bulletin of Spanish Studies 89 (4), 575-596.

NTLE: Lidio Nieto Jiménez y Manuel Alvar EzquerRa, 2007: Nuevo tesoro lexicográfico del español (5. XIV-1726), Madrid: Arco/Libro.

PADEN, Jeremy, 2007: "The Iguana and the Barrel of Mud: Memory, Natural History, and hermeneutics in Oviedo's Sumario de la Natural Historia de las Indias", Colonial Latin American Review 16 (2), 203-226.

Quirós Garcia, Mariano, y José Luis Ramirez Luengo, 2015: "Observaciones sobre el léxico del español de Yucatán (1650-1800)”, Revista de Filología Española 95(1), 183-210.

Turpin, Coll. D., 1987: "The Origin of Common Spanish Names for Fifteen Well-known Plants of Mexico", Names 35(2), 74-85.

Zamora Munné, Juan Clemente, 1976: Indigenismos en la lengua de los conquistadores, Puerto RiCO: UPREX. 